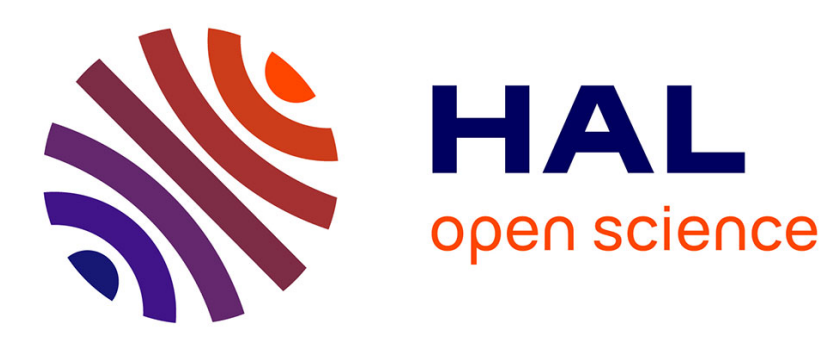

\title{
The design of TaCS: Applying social tagging to enhance learning
}

Elise Lavoué

\section{To cite this version:}

Elise Lavoué. The design of TaCS: Applying social tagging to enhance learning. International Journal of Learning Technology (IJLT), 2012, 7 (3), pp.314-330. hal-00731092

\section{HAL Id: hal-00731092 \\ https://hal.science/hal-00731092}

Submitted on 12 Mar 2015

HAL is a multi-disciplinary open access archive for the deposit and dissemination of scientific research documents, whether they are published or not. The documents may come from teaching and research institutions in France or abroad, or from public or private research centers.
L'archive ouverte pluridisciplinaire HAL, est destinée au dépôt et à la diffusion de documents scientifiques de niveau recherche, publiés ou non, émanant des établissements d'enseignement et de recherche français ou étrangers, des laboratoires publics ou privés. 


\title{
The design of TaCS: applying social tagging to enhance learning
}

\author{
Elise Lavoué \\ Université de Lyon, CNRS \\ Université Jean Moulin Lyon 3, MAGELLAN, LIRIS, UMR5205 \\ 6 cours Albert Thomas BP 8242 \\ 69355 Lyon Cedex 08, France \\ Elise.Lavoue@univ-lyon3.fr
}

\begin{abstract}
We describe a web-based educational platform relying on the use of tags as learning resources in a social context. Tags are user-generated keywords used to organise and improve access to online information resources. Social tagging refers to the practice of labeling or categorising resources in a shared online environment. Our platform, named TaCS (Tag-based Collaborative System), is a first attempt at implementing a recent theoretical framework specific to social tagging that defines learning and knowledge building as a co-evolution of cognitive and social systems. Within this framework, design choices have been made to support individual, social and collaborative learning processes. To illustrate the usability of TaCS, we describe an example of its integration in a real course during 5 weeks. Observed uses and opinions expressed by students tend to validate the implementation of the theoretical framework, and the value of $\mathrm{TaCS}$ as an educational platform.
\end{abstract}

Keywords: TaCS; tagging; social learning; web-based system; educational technology design; collaborative learning.

Biographical notes: Élise Lavoué is an Associate Professor in Information Systems in the IAE Lyon, the University of Lyon School of Management, and at the MAGELLAN Research Centre. She is also a collaborator of the LIRIS Laboratory, SILEX team. She received an Industrial Engineering degree in 2005 and a Doctoral Thesis in Computer Science in 2008 from the INSA Lyon (National Institute of Applied Sciences, Engineering University), France. There she developed the TE-Cap environment to connect communities of Practice of tutors in university. She joined the IAE Lyon in 2009. Her research interests include several topics: Collaborative Information Systems, Collective Knowledge Management and Assistance to Human Tutoring, in the fields of Technology Enhanced Learning (TEL), Computer Supported Collaborative Learning (CSCL) and Knowledge Engineering. 


\title{
The design of TaCS: Applying social tagging to enhance learning
}

\begin{abstract}
We describe a web-based educational platform relying on the use of tags as learning resources in a social context. Tags are user-generated keywords used to organise and improve access to online information resources. Social tagging refers to the practice of labeling or categorising resources in a shared online environment. Our platform, named TaCS (Tag-based Collaborative System), is a first attempt at implementing a recent theoretical framework specific to social tagging that defines learning and knowledge building as a co-evolution of cognitive and social systems. Within this framework, design choices have been made to support individual, social and collaborative learning processes. To illustrate the usability of TaCS, we describe an example of its integration in a real course during 5 weeks. Observed uses and opinions expressed by students tend to validate the implementation of the theoretical framework, and the value of TaCS as an educational platform.
\end{abstract}

Keywords: tagging; social learning; web-based system; educational technology design; collaborative learning.

\section{Introduction}

Social tagging is the activity of annotating and classifying digital resources with keywords (tags as metadata). Nowadays social tagging is used by most web-based information systems for the collaborative indexing of massive amount of information (Yeung, Gibbins, \& Shadbolt, 2009). However, little is known about how Web 2.0 technologies, particularly social tagging, may directly interact with individuals at the knowledge and cognitive level (Held \& Cress, 2009). Some recent work (Fu, 2008; Kimmerle, Cress, \& Held, 2010) showed how social tagging supports cognitive and social learning processes. Based on these results, we were interested in the use of tagging in education as a support for enhancing learning experience and fostering group dynamics in collaborative activities. This paper describes the design of the Tag-based Collaborative System (TaCS) we developed. This system aims to enhance individual, social and collaborative learning processes for learners involved in a common course, particularly during information seeking activities.

We first describe in section 2 our approach of tagging as a process that occurs in a socio-technical context. We then discuss the Kimmerle et al.'s theoretical framework (2010), which defines learning and knowledge building as a co-evolution of cognitive and social systems during tagging activities by users. Based on this framework, we describe in section 3 the TaCS platform and explain which learning processes are expected to be associated with its use. As an example of integration of TaCS in a course, we report in section 4 on an educational scenario carried out by 17 students during 5 weeks. We present the main observed uses and opinions expressed by students. This exploratory study illustrates the possibilities of TaCS and its value as an educational platform. 


\section{Tagging for Learning}

In this section, we first define folksonomy and social tagging, and position our works among current research topics related to these terms. We then study existing systems based on tags to show that they are rarely developed to enhance learning. We finally describe the learning processes that occur during tagging activities, and upon which is based the design of TaCS described in section 3. We finally show how the tagging process in a social context can be used to enhance learning.

\subsection{Folksonomies and social tagging}

Tags are user-generated keywords used to describe, organise and improve access to online information resources. The resulting assemblage of tags forms a folksonomy: a conflation of the words folk and taxonomy (Dahl \& Vossen, 2008) used to refer to an informal assemblage of related terminology. Tagging is a means to support personalised use of networked resources: users can associate their own keywords with on-line resources for personal indexing and retrieval of information. Social Tagging refers to the practice of publicly labelling or categorising resources in a shared, on-line environment (Trant, 2009).

Published studies about social tagging and folksonomy focused on three broad approaches: folksonomy itself, the nature of social tagging systems, and tagging (Trant, 2009). Firstly, we can think of folksonomy as the resulting collective vocabulary (with a focus on knowledge organisation). This approach is interested in the role of tags in indexing and information retrieval with respect to the following issues: vocabulary analyses, control and evolution of folksonomy (Guy \& Tonkin, 2006), finding structures in folksonomy (Kipp \& Campbell, 2006) and comparing them with ontology (Mika, 2007). This approach is out of the scope of our study.

Secondly, we can think of tagging as a process and be interested in the behavior of users. This type of research focuses on what users do when they tag. Existing studies in this field are quite preliminary, and mostly reported basic descriptive statistics. Since tagging systems are relatively new, understanding what users are doing with them helps developing an appreciation of their contribution to the digital information landscape (Trant, 2009). In an individual perspective, the need to organise information for later personal retrieval has been found as one of the most important motivation for tagging (Gao, 2011). In a social perspective, some recent work (Fu, 2008; Kimmerle et al., 2010) suggested that social tagging supports cognitive and social learning processes. Our research, described in details in section 2.3, is based upon these results.

Thirdly, we can think of social tagging as a socio-technical context within which tagging takes place. Studies in this field describe systems that use tags and the interrelationships of their parts. They mainly focus on two aspects: information discovery and searching using tags.

For the design of TaCS, we were mainly interested in two aspects of social tagging systems:

- Visualisation: the most prevalent visualisation of tags used is the tag cloud, a visualisation of the tags represented in a system with tags weighted based on frequency of occurrence. Bateman, Gutwin and Nacenta (2008) show that weights associated to size and font have the strongest effect on users. 
- Ranking: the availability of tag data linked to a particular user enables ranking of users, based on the characteristics of their tagging (Trant, 2009). Michlmayr and Cayzer (2007) studied these characteristics in order to make the users' expertise explicit through the visualisation of users' tags space. Yew, Gibson and Teasley (2006) show that shared tagging of content can also be helpful for group formation, and for knowledge sharing.

As our works focuses on the use of tagging to enhance learning processes, we describe in more details tag-based systems used in the educational area in the next section. .

\subsection{Tag-based educational systems}

Social tagging systems are usually used to facilitate the collaborative indexation of massive quantity of information and improve their accessibility (Millen, Feinberg, \& Kerr, 2006). Connotea ${ }^{1}$ and CiteULike ${ }^{2}$ are examples of online reference management and social bookmarking services for assisting scientists, researchers and academics in storing, organising, sharing, and discovering links to academic scientific and research papers. Tag clouds are also used as an indexation and search tool by communities of teachers, as in the Cloudworks Web site (Conole \& Culver, 2010), created for teachers to discuss their practices and ideas about educational design. The bookmarks sharing tool SemanticScuttle (Huynh-Kim-Bang, Dané, \& Grandbastien, 2008) also helps communities of teachers structuring their tags by creating explicit inclusion and synonymy relationships between tags.

The ASK - LOST 2.0 system (Kalamatianos, Zervas, \& Sampson, 2009) uses tags to index all types of digital educational resources (images, videos, texts, URL). Dahl and Vossen (2008) introduced the term "learning folksonomy" and applied social tagging to real-world applications: the metadata repository share.loc and the content repository Learnr.

Some authors have used tags for other educational applications. The social annotation and tagging systems OATS (Bateman, Brooks, Mccalla, \& Brusilovsky, 2007) and SparTag.us (Nelson et al., 2009) allow highlighting and tagging only part of a text. For every annotated text, users can see their tags as well as the most frequent tags given by other users. It is also possible to see the cloud of the most used tags. However, these tools do not allow collaborative work, since every student annotates the text individually.

The online collaborative system TACO (Chen, Chen, \& Sun, 2010), based on tags, was developed to support language learning. This system was designed to improve the understanding of written English and, more precisely, to favour development of critical thinking capacities. A forum is linked to each tag to allow learners to criticise them and to exchange ideas (all tags are visible for all learners). This system especially aimed at helping teachers evaluating more precisely their students through the use of an automatic scoring mechanism applied on their tags.

In conclusion, usually only the community effect of tags (the use by a large number of users) has been used used for the indexation and the search for information. Only the TACO environment was really designed for collaborative learning, with negotiation and criticism of tags. However TACO is very specific to language learning. We present in the

\footnotetext{
${ }^{1} \mathrm{http}: / / \mathrm{www}$. connotea.org/

$2 \mathrm{http} / / / w w w . c i t e u l i k e . o r g /$
} 
next section a more generic framework for the learning processes occurring when users tag in a social context.

\subsection{Learning processes involved in tagging}

Within the framework of Cognitive Distributed Systems (DCS), Fu (2008) studied the interactions between the internal and external representations of concepts, tags and documents when a user is engaged in iterative explore-and-comprehend cycles. Their results suggest that (1) the interactions between internal concepts and external tags gradually lead to the sharing and assimilation of conceptual structures and (2) social tagging systems are then a means for social exchange of knowledge structures. Based on this work, Kimmerle et al. (2010) presented a model that defines learning and knowledge building as a co-evolution of cognitive and social systems while users tag. Their theoretical framework distinguishes four processes:

- Externalisation: learners externalise their knowledge about a resource by assigning tags to it. To create tags, users have to articulate their own cognitive concepts and to translate them into keywords. This cognitive effort stimulates information processing in such a way that individual learning may occur (Budiu, Pirolli, \& Hong, 2009).

- Internalisation: by navigating within the information space using the tag clouds, users collect information related to a tag. On the one hand, they learn tags used by others and, as a consequence, how others classify their resources. On the other hand, tags show new interconnections between concepts for users. This can lead to the incorporation of the concepts of the community and to the modification of the individual cognitive structures of users.

- Assimilation: by discovering and using new tags (and the associated concepts) that are in agreement with their knowledge, users can widen their knowledge but do not develop new, different concepts.

- Accommodation: users can question and modify their cognitive concepts by learning that their associations on a specific domain are rather different, inadequate, or even false. This can happen when users realise that other users use tags that are very different from theirs, which implies that specific resources or tags can be bound to very different concepts.

Based on the framework detailed above, we identified several concepts related to social tagging:

- A tag: an external representation of a cognitive concept in a domain.

- A tag-document relationship: a representation of an explicit conceptual relationship between a document and a cognitive concept.

- An individual folksonomy: a simplified external representation of learners' cognitive concepts in a domain.

- A group folksonomy: a shared external knowledge structure of the group related to a domain.

The design of TaCS is based on this theoretical framework and its functionalities aim to enhance the learning processes described above. 
Author

\section{TaCS: A tag-based collaborative system for learning}

In this section, we describe the Tag-based Collaborative System (TaCS) (Lavoué, 2011a; Lavoué, 2011b) we have developed to implement the theoretical framework described in section 2.3. TaCS is based on the content management system Joomla! ${ }^{1}$. We integrated existing components that we modified and we also developed a specific component to offer the functionalities related to tags.

TaCS is meant to support the collaborative activity of information seeking for learners involved in a common course to learn the concepts of a same domain. Three types of actors are supported on TaCS: the individual learner, the group who carries out collaborative activities and the class composed of all the learners who use TaCS in a course. A virtual space is offered to each actor with its own functionalities.

\subsection{Individual learning on TaCS}

\subsubsection{Creation of documents and tags}

Learners can create and manage their own documents and tags from their personal space on TaCS (see Fig. 1). A document is a text with several associated information: its title, its reference (the file or the link of the Web page where the contents were extracted), and its assigned tags. Tags are keywords created by a user when s/he creates or edit a document on TaCS. A learner can assign as many tags as s/he wants to his/her documents to identify its main concepts or ideas. S/he has to assign at least one tag.

Figure 1. Creation of a document and its assigning tags

According to the learning processes described in section 2.3, we consider that the association of a tag with a document represents an explicit conceptual relationship: the learner uses the tag for example to describe and/or to summarise the document. According to the externalisation process, we think that the difficulty in assigning tags to documents can bring learners to question the relevance of the document with regard to the domain studied. According to Bateman et al. (2007), referring to the Bloom's Taxonomy of Learning (Bloom, 1979), "learners who use tags show evidence of moving up the hierarchy from the lower "consumption"- based levels of learning (knowledge and comprehension) to higher levels of applied and metacognitive knowledge (application and analysis)." So, we expect learners to think about the documents, to understand their content and to apply their synthesis skills by identifying keywords (tags) that summarise the main ideas and concepts of the document.

\subsubsection{Visualisation of individual tag clouds}

A learner's folksonomy is automatically composed of all the tags that $\mathrm{s} / \mathrm{he}$ has created. The folksonomy is represented on the shape of a tag cloud. For each tag, the number of times it has been used is indicated (the same tag can be associated with several documents). The more a tag is used, the bigger its size and its font weight are in the tag cloud (see Fig. 2). These individual tag clouds can be considered as simplified representations of the cognitive concepts of learners related to a subject. By giving

\footnotetext{
${ }^{1}$ http://www.joomla.org/
} 
learners the possibility to visualise their own tag clouds, we aim at bringing them having a reflexive approach and to question their appreciation of the domain under study. For example, based on the shape of tag clouds, they can detect a gap between the approach they think they have and the external representation of their cognitive concepts in the domain.

On a same interface on TaCS (see Fig, 2), learners can see the cloud of the tags they currently use to describe documents, the cloud of the tags they used to describe documents but they have deleted (deletion of a tag does not imply deletion of the associated document) and the cloud of the tags they used to navigate on the platform. This distinction is based on the recommendations of Glahn, Specht and Koper (2008) who emphasise the importance for reflection support to consider and distinguish implicit information and explicit information in tags, as they relate to different types of user actions and user interests. For the design of TaCS, we considered that the implicit information (deleted tags and tags used to navigate) reflects for learners an image of their actions: they can come to realise, for example, that they often use a tag to navigate while they never used it to describe a document. The cloud of deleted tags is a "memory of the tags" as learners can discover that they use a tag they have already deleted several times.

Figure 2. Tag clouds of a learner on TaCS (implicit and explicit information)

\subsection{Social learning on TaCS}

\subsubsection{Comparison of the individual and collective tag clouds}

The TaCS platform aimed at supporting collaborative activities carried out by groups of learners, more specifically the search for information related to a domain. The platform can gather hundreds of learners involved in the same course (an example is given in section 4). All learners registered on TaCS are called "The class". In a collective activity, the tag cloud of a group is automatically composed of all tags created by members of this group. The tag cloud of the class is composed of all tags created on TaCS by all users. In these two levels of tag cloud, the frequency of occurrence of each tag depends on its frequency in each individual folksonomy.

The learners can visualise the tag cloud of their group on the "Group space" and the tag cloud of all the learners on the "Class space" (see Fig. 1). According to the assimilation process defined for a social context, by discovering and using new tags (and the associated concepts) in the collective tag clouds, we think that learners can widen their knowledge about a domain. To facilitate the assimilation process, the TaCS platform offers several functionalities to visually compare the individual and collective tag clouds of learners (name and degree of use of tags):

- A visual comparison on a same interface of the individual learners' tag cloud with the tag cloud of their group (see Fig. 3).

- A visual comparison on a same interface of the tag cloud of their group with the global tag cloud of the class (all the learners).

According to Bateman et al. (2007), "reviewing of tags (i.e. comparing tags used by a community of taggers) would potentially facilitate the move to the highest levels of Bloom's Taxonomy of Learning (synthesis and evaluation)". We consider an individual 
tag cloud to be an external representation of the learners' cognitive concepts and a collective tag cloud to represent the shared external knowledge structure of the group. By allowing learners to compare "at a glance" these two representations, we aim to enhance the assimilation of new concepts, and therefore to bring learners to create and/or delete documents and/or tags.

Figure 3. Comparison on TaCS of the tag cloud of a student and the tag cloud of his/her group

\subsubsection{Visualisation of tag-documents and tag-users relationships}

We agree with Chen et al. (2010), who state that tags can facilitate the development of a learning community by building a collective identity among users. When clicking on a document or a tag, learners have access to its details (see Fig. 4). The details are:

- For a tag: learners that have used it, associated documents and their definition. These definitions can be created collaboratively by all the members of a group in a contextual forum (see section 3.3).

- For a document: its title, its date of creation, its text, its references and the assigned tags.

Figure 4. Details of tags and documents: document-tags, tag-documents and tag-users relationships

A study conducted by Nelson et al. (2009) suggests that expertise delivered through social annotation mechanisms (users have access to others' annotations) is helpful to users in learning unfamiliar domains. In agreement with Glahn et al. (2008), we think that implicit relationships can be useful for social learning processes, especially internalisation and accommodation processes. According to the accommodation process, we think that the visualisation of explicit tag-document relationships (tags assigned to document, documents associated with a tag by learners in the same group) can bring learners to question their own conceptual relationships and, possibly, to modify them. Furthermore, according to the internalisation process, by collecting information on a tag, learners can discover new interconnections between concepts and therefore incorporate the concepts of the community and modify their individual cognitive structure. These learning processes can lead to the creation of documents bound to a tag and/or to the association of new tags to a document.

\subsection{Collaborative learning on TaCS}

In addition to the social learning processes supported by TaCS and described in previous sections, we think that tags could also enhance negotiation and criticism skills in the context of collaborative activities. TaCS works according to the following principle: the set of documents of a learning group is composed of all the documents proposed by the members individually, and the tag cloud of a group is composed of all the individual folksonomies of learners as well. Only their author can delete documents and tags and add tags to a document. So learners of a group have to share their expertise on the domain and to negotiate to reach agreement.

To support exchanges, a contextual forum is linked to each tag. The learning groups also have a dedicated forum, used for example to discuss their documents. By participating on a forum, learners from a group are able to criticise the contributions of 
other learners and to negotiate in order to reach agreement about the tag cloud of the group. As there is no automatic deletion of tags, users also have to discuss synonymy and other vocabulary problems related to tags, so as to keep only those relevant for the learning group. These discussions about tags can facilitate the assimilation of the associated concepts.

The forum is also meant for a specific collaborative activity: creation of a common definition of a tag by all members of a learning group (see section 4.1). This collaborative learning activity can cause learners sharing their knowledge, expertise and interest about the concept related to a tag.

\section{An example of integration of TaCS in an educational scenario}

This section is dedicated to the report of a case study of the integration of TaCS in a course, aimed at bringing practical experience to practitioners who would like to use $\mathrm{TaCS}$ in their teaching. We first describe an educational scenario carried out by 17 students during 5 weeks. We then report on the observed students' uses of TaCS during the scenario and their opinion about TaCS. The results tend to validate the implementation of the theoretical framework of Kimmerle et al. (2010), and the value of $\mathrm{TaCS}$ as an educational platform.

\subsection{An educational scenario with TaCS}

We integrated TaCS according to an educational scenario in a course about "Collaborative Information Systems" of a 5th year in University, from 11 November 2010 to 16 December 2010. The students were gathered by groups of 4 or 5 to study the case of a French company, which organises events in France and abroad. They had to design a collaborative information system to facilitate the collaborative work of the employees of this company. The students were asked to search for information upon which they could base their study. They were assessed based on their final set of documents and tag clouds. The students had access to the TaCS functionalities according to five phases to carry out individual and collaborative activities. Each phase, described below, lasted a week.

- Phase 1: Individual creation of documents and tags

At first, the students were asked to search individually for interesting documents related to the case study and to assign tags to them (at least one). The texts of the documents were extracted from any online resources. The students themselves created the tags. Learners had access to their individual space to manage their documents and to visualise their own tag clouds.

- Phase 2: Comparison of the individual tag clouds and the learning group's tag clouds

During this individual activity, the learners had access to the tags created by other members of their group. We encouraged learners to compare their tags with the tags of their group, to modify their own tags and/or to search for new documents.

- Phase 3: Document sharing and negotiation within the learning group 


\section{Author}

The students had access to all the documents of their group on the group space. They were able to discuss the documents through the group forum. We asked learners to keep only the documents they estimated relevant to the case study.

- $\quad$ Phase 4: Tag sharing and negotiation within the learning group

Learners were asked to create a common tag cloud for their group, describing in the best possible way its approach to the case study. For that purpose, the group had to agree on the tags through discussion on the contextual forums associated with the tags. Furthermore, learners had to reach a common definition for every tag of the group. We expected learners to define and discuss the meaning of the tags. Learners could see for each tag the learners that had used it and its associated documents.

- Phase 5: Comparison of the learning group's tag clouds and the tag clouds of all learners

Finally, students had access to the tag clouds of the class, automatically generated from individual tags, to compare it with the tag clouds of their learning group. Each group could modify its documents and assigned tags. After having agreed on a final set of documents, students were asked to agree on a final tag cloud for their group, which represents the shared external knowledge structure of the group related to the case study.

\subsection{The observed students' uses and opinions of TaCS}

We summarise in this section (1) the main observed students' uses of TaCS during the scenario and (2) the learners' opinions and explanations regarding the used or unused functionalities and the learning processes they think they applied. The data were collected through the analysis of use tracks and a questionnaire composed of 47 questions filled anonymously and freely at the end of the course. The results are reported according to the learning processes described in section 3.

We specify that 13 learners filled in the questionnaire. We identify several explanations for the 4 students who did not fill in it. Mainly, some students did not take the time to answer the questionnaire, which was distributed at the end of the course. Moreover, although the questionnaire was anonymous, we think that some students were afraid that their responses have an influence on their final grade for the course. As the questionnaire was anonymous, we cannot analyse the use of TaCS by the students who did not fill in it. We therefore cannot conclude on the influence of non-filled in questionnaires on the results presented in this section.

\subsubsection{General results}

We observed that learners created 201 documents, with an average of 12 documents per learner. A considerable proportion of the created documents was deleted at the end of the scenario (61 of the 201 documents created; the 4 groups deleted respectively $30.6 \%, 70.4$ $\%, 73.5 \%$ and $11.6 \%$ of its documents).

Learners created 354 different tags and 969 tag-document relationships (tags assigned to documents), with an average of 32 distinct tags per student (the same tag can be used by several students) and approximately 5 tags (precisely 4.8) per document. The students revised their tags during the study: 251 of the 354 created tags were deleted at the end of the scenario. On average, learners deleted 19 tags. 407 of the 969 created relationships were deleted at the end of the scenario. 
All the final tags and documents were considered relevant for the case study by the teacher who assessed the learners individually and by group at the end of the course. The final assessment of the students was therefore based both on the number of tags and documents created by the students and on their participation in the forums to define the tags.

\subsubsection{Individual learning}

The individual phase 1 of the scenario was meant to enhance reflection activities during creation of documents and their associated tags according to an externalisation process (see section 3.1). This is confirmed by the fact that much of the learners thought that assigning tags to documents helped in understanding the documents themselves ( 8 yes, 4 no and 1 nonresponse). The observed uses strengthen this result, since we notice that almost all documents were created and deleted during the first phase (see Fig. 5): the students created 180 documents (among the 201 final documents) and deleted 57 documents (among the 61 deleted documents during the study). We also observed that the majority of the tags (respectively $65.0 \%, 76.3 \%, 79.6 \%$, and $86.5 \%$ ) and relationships (respectively $57.9 \%, 76.4 \%, 67.4 \%$, and $74.0 \%$ ) were created during the first phase. Furthermore, the majority of the students thought that their final tag cloud reflected adequately the main ideas and concepts of their documents and of their own approach to the case study (see Table 1). Indeed, it seems quite natural that users created and deleted a considerable proportion of the tags and documents in the first phase of the study due to the scenario itself.

Figure 5. Documents, tags and tag-document relationships created and deleted at each stage of the scenario

Table 1. Response of the students on their satisfaction with the reflection by their tags of: (1: low satisfaction; 4: high satisfaction)?

\begin{tabular}{|c|c|c|c|c|}
\hline & 1 & 2 & 3 & 4 \\
\hline The main ideas and concepts of their documents & $0.0 \%$ & $0.0 \%$ & $\mathbf{7 6 . 9} \%$ & $\mathbf{2 3 . 1} \%$ \\
\hline Their own approach of the case study & $7.7 \%$ & $15.4 \%$ & $\mathbf{6 1 . 5} \%$ & $\mathbf{1 5 . 4} \%$ \\
\hline
\end{tabular}

\subsubsection{Social learning}

Social learning (see section 3.2) was expected to occur during phase 2, through visual comparison of the individual tags and the tag clouds of the learning group, and during the phase 5, through visual comparison of the tag clouds of the learning group and that of all users. During phase 2, the learners also had access to the details of the tags and documents and so could learn tag-document and tag-user relationships.

Regarding phase 2 (see Fig. 5), the students thought that the comparison of their own tags with those of their group mainly allowed them to have a view of the others' work and led them to question themselves and to make their tags evolve (see Table 2). Another point that confirms this tendency is that students also thought that they made their tags evolve mainly for two reasons (see Table 3): after comparison with the tags of the members of their group and through a collective decision of the group (this second point is discussed in section 4.2.4). These results are strengthened by the fact that a significant proportion of the tags and tag-document relationships was created during phase 2. 
Author

Table 2. Response of the students on the perceived effect of the comparison of their own tags with those of their group (1: low effect; 4: high effect)

\begin{tabular}{|c|c|c|c|c|}
\hline & 1 & 2 & 3 & 4 \\
\hline It allows them to have a view of the others' work & $0.0 \%$ & $23.1 \%$ & $30.8 \%$ & $\mathbf{4 6 . 2} \%$ \\
\hline It led them to question themselves & $7.7 \%$ & $23.1 \%$ & $\mathbf{4 6 . 2} \%$ & $23.1 \%$ \\
\hline They were surprised & $23.1 \%$ & $\mathbf{5 3 . 8} \%$ & $23.1 \%$ & $0.0 \%$ \\
\hline It led them to make their tags evolve & $7.7 \%$ & $30.8 \%$ & $\mathbf{3 8 . 5} \%$ & $23.1 \%$ \\
\hline It led them to make their documents evolve & $\mathbf{3 8 . 5} \%$ & $15.4 \%$ & $\mathbf{2 3 . 1} \%$ & $\mathbf{2 3 . 1} \%$ \\
\hline
\end{tabular}

Table 3. Reasons why the respondents questioned and made their own tags evolve

\begin{tabular}{|c|c|c|}
\hline & Yes & No \\
\hline After comparing with the tags of the members of their group & $\mathbf{6 9 . 2} \%$ & $30.8 \%$ \\
\hline After better understanding the case study & $23.1 \%$ & $\mathbf{7 6 . 9 \%}$ \\
\hline After reading new documents & $23.1 \%$ & $\mathbf{7 6 . 9} \%$ \\
\hline After visualising the tags of the other groups & $30.8 \%$ & $\mathbf{6 9 . 2} \%$ \\
\hline By choice of the group (collective decision) & $\mathbf{6 9 . 2} \%$ & $30.8 \%$ \\
\hline
\end{tabular}

Regarding the phase 5 (see Fig. 5), few students reported to have made their tags evolve after visualising the tags of the class (all the learners) (see Table 3). Furthermore, respondents answered that they had looked at the tags proposed by other groups ( 9 yes, 3 no, 1 nonresponse) but that it had not significantly influenced them ( 2 yes, 6 no and 5 nonresponses). Few students looked at the documents proposed by other groups (4 yes, 8 no and 1 nonresponse) and for those who looked at them, this did not influence them. These results are strengthened by the fact that only two groups (among the 4 groups involved) made their tags evolve during phase 5 (they deleted tags) and this evolution was rather limited. This result may be due to the educational scenario itself, which could have affected the learners' behavior. As they could discuss the documents and tags of their group during phases 3 and 4 in order to reach an agreement, they may not have wanted to discuss again after visualising the tag clouds of the class. Maybe the results would have been different if learners had had access to the tags of all the learners of the class before discussing collaboratively the tags and documents of their learning group.

\subsubsection{Collaborative learning}

Collaborative learning was expected during the collaborative activities of phases 3 and 4 , especially through discussions within the learning group on its forum (phase 3) and on the contextual forums associated with the tags to be defined (phase 4).

As a general result for these two phases, the students thought that they applied the following collaborative skills: criticism of the other members' submissions (documents and tags) (11 yes, 1 no and 1 nonresponse); negotiation of tags (10 yes, 1 no and 2 nonresponses), negotiation of the definition of tags (10 yes and 3 no) and documents (11 yes, 1 no and 1 nonresponse). Furthermore, the students were satisfied with the coherence of the tag cloud and of the set of documents of their group, as well as with the image of their approach of the case study they reflected (see Table 4), although the number of tags and relationships deleted by each one is rather high (62\% of the distinct tags and $42 \%$ of the tag-document relationships). 
Table 4. Response of the students on their satisfaction with the following statements: (1: low satisfaction; 4: high satisfaction)?

\begin{tabular}{|c|c|c|c|c|c|}
\hline & 1 & 2 & 3 & 4 & $\begin{array}{l}\text { nonres } \\
\text { ponses }\end{array}$ \\
\hline $\begin{array}{l}\text { The tag cloud of their group reflects their own } \\
\text { approach of the case study }\end{array}$ & $7.7 \%$ & $7.7 \%$ & $61.5 \%$ & $23.1 \%$ & $0.0 \%$ \\
\hline The tag cloud of their group is coherent & $0.0 \%$ & $0.0 \%$ & $46.2 \%$ & $46.2 \%$ & $7.7 \%$ \\
\hline $\begin{array}{l}\text { The tag cloud of their group reflects its } \\
\text { approach of the case study }\end{array}$ & $7.7 \%$ & $0.0 \%$ & $30.8 \%$ & $46.2 \%$ & $15.4 \%$ \\
\hline $\begin{array}{l}\text { The set of documents of their group is } \\
\text { coherent }\end{array}$ & $0.0 \%$ & $0.0 \%$ & $61.5 \%$ & $30.8 \%$ & $7.7 \%$ \\
\hline $\begin{array}{l}\text { The set of documents of their group reflects its } \\
\text { approach of the case study }\end{array}$ & $15.4 \%$ & $7.7 \%$ & $46.2 \%$ & $23.1 \%$ & $7.7 \%$ \\
\hline
\end{tabular}

However, we expected that students would revise their documents during the collaborative phases and it is surprising to observe that the comparison of their documents with those of their group had no influence on the evolution of the documents (creation or deletion). Phase 3 also had little influence on the creation and the deletion of tags and their relationships to documents (see Fig 5.). So we conclude that the documents alone are not sufficient resources to enhance collaborative learning processes.

A significant part of the tags and their relationships to documents was created during the collaborative phase 4 (see Fig. 5), during which learners had access to the tag clouds of their group in addition to its documents. We observed that most of tags (respectively $74.0 \%, 81.5 \%, 68.0 \%$ and $97.3 \%$ of the whole deleted tags) and tag-document relationships (respectively $74.0 \%, 84.6 \%, 61.6 \%$ and $97.7 \%$ of the whole deleted relationships) have also been deleted during the phase 4. According to the design of $\mathrm{TaCS}$, only the creator of a document can associate it with a tag. So we deduce that the negotiation within the group led students to modify their tags and tag-document relationships. This point is strengthened by the answers of the students in table 3, which show that they made their tags evolve mainly by a collective decision of the group on the associated contextual forums. However, this interpretation has limits: the assignment included reaching a common definition of every tag; this perhaps led to a desire to delete tags in phase 4. Among the 103 final tags, 89 tags have been defined, all during the phase 4.

Regarding the use of the forums, 16 students wrote 274 messages on the contextual forums associated with tags, whereas 10 students wrote only 36 messages on the forums of the group during the study. These results highlight the relevance of contextual forums associated with tags, for example to negotiate their meaning. A possible improvement would be to associate a forum with each document, as provided for tags, to enhance the discussions on documents.

\section{Conclusion and future work}

In this paper, we describe the web-based learning platform TaCS, which is designed to support several learning processes involved in a social tagging process. Our platform is a first attempt at implementing a recent theoretical framework specific to social tagging that defines learning and knowledge building as a co-evolution of cognitive and social systems. We distinguish three types of learning processes: 
Author

- Individual learning (1) by an externalisation process when users create tags and associate them with documents and (2) by a reflexive process when learners visualise their own tag clouds.

- Social learning (1) by an assimilation process when users visually compare their own tag clouds with the collective tag clouds of their group or the class and (2) by accommodation and internalisation processes when users discover tag-document and tag-user relationships.

- Collaborative learning when learners negotiate within their learning group by using the forums (1) to reach agreement on the set of documents and the tag clouds of the group and (2) to define a common definition of tags.

We then reported on an example of educational scenario carried out by using the TaCS platform, which illustrated its usability and validated the implementation of the theoretical framework. We showed that the observed uses are consistent with the expected uses. Furthermore, the opinions expressed by students are in agreement with the expected learning processes. These first results are positive and show the interest of TaCS as an educational platform. However, the case study reported in this paper is an exploratory study that cannot allow us to make conclusions on the precise effects of using TaCS on learning. We are currently considering a number of studies with more students to experimentally validate our approach and more precisely to study the effect of using each of the functionalities on specific learning processes.

This first study allowed us to identified two possible improvements in a short-term: a better integration of the class (the community of learners) in the educational scenario and the association of contextual forums with the documents to improve the negotiation of documents within the learning groups. More generally, the TaCS platform offers new opportunities for educational activities, not only for learners but also for teachers. For instance, we think that the learners' tag clouds can be used as indicators to reflect the learners' acquisition of the concepts of a domain and the learning processes they applied. We will develop monitoring functionalities based on tag clouds to help teachers to monitor learners' activities and evaluate them (individually and collectively). Tags will also be used as metadata on learners to help teachers to personalise the learning activities.

\section{References}

Bateman, S., Brooks, C., Mccalla, G., \& Brusilovsky, P. (2007). Applying Collaborative Tagging to E-Learning. Proceedings of the 16th International World Wide Web Conference (WWW2007). Banff, Alberta, Canada.

Bateman, S., Gutwin, C., \& Nacenta, M. (2008). Seeing things in the clouds: the effect of visual features on tag cloud selections. Proceedings of the nineteenth ACM conference on Hypertext and hypermedia (p. 193-202). Pittsburgh, PA, USA: ACM.

Bloom, B. S. (1979). Taxonomy of educational objectives: the classification of educational goals. Handbook I, Cognitive domain (Vol. 1-1). London: Longman.

Budiu, R., Pirolli, P., \& Hong, L. (2009). Remembrance of things tagged: how tagging effort affects tag production and human memory. Proceedings of the 27th international conference on Human factors in computing systems (p. 615-624). Boston, MA, USA: ACM. 
Chen, J.-M., Chen, M.-C., \& Sun, Y. S. (2010). A novel approach for enhancing student reading comprehension and assisting teacher assessment of literacy. Computers \& Education, 55(3), 13671382 .

Conole, G., \& Culver, J. (2010). The design of Cloudworks: Applying social networking practice to foster the exchange of learning and teaching ideas and designs. Computers \& Education, 54(3), 679-692.

Dahl, D., \& Vossen, G. (2008). Evolution of learning folksonomies: social tagging in e-learning repositories. International Journal of Technology Enhanced Learning, 1(1/2), 35 - 46.

Fu, W.-T. (2008). The microstructures of social tagging: a rational model. Proceedings of the ACM conference on Computer Supported Cooperative Work (CSCW 2008) (p. 229-238). San Diego, CA, USA: ACM.

Gao, Q. (2011). An Empirical Study of Tagging for Personal Information Organization: Performance, Workload, Memory, and Consistency. International Journal of Human-Computer Interaction, 99999(1), 1.

Glahn, C., Specht, M., \& Koper, R. (2008). Implications of Writing, Reading, and Tagging on the Web for Reflection Support in Informal Learning. Proceedings of the 3rd European conference on Technology Enhanced Learning (EC-TEL 2008) (p. 110-121). Maastricht, The Netherlands: Springer-Verlag.

Guy, M., \& Tonkin, E. (2006). Folksonomies. Tidying up Tags? D-Lib Magazine, 12(1).

Held, C., \& Cress, U. (2009). Learning by Foraging: The Impact of Social Tags on Knowledge Acquisition. Proceedings of the 4th European conference on Technology Enhanced Learning (ECTEL 2009) (p. 254-266). Nice, France.

Huynh-Kim-Bang, B., Dané, E., \& Grandbastien, M. (2008). Merging semantic and participative approaches for organising teachers' documents. Proceedings of ED-Media 08 - World Conference on Educational Multimedia, Hypermedia \& Telecommunications (p. 4959-4966). Vienna, Austria.

Kalamatianos, A., Zervas, P., \& Sampson, D. G. (2009). ASK-LOST 2.0: A Web-Based Tool for Social Tagging of Digital Educational Resources. Proceedings of the Ninth IEEE International Conference on Advanced Learning Technologies (ICALT 2009) (p. 157-159). Riga, Latvia: IEEE Computer Society.

Kimmerle, J., Cress, U., \& Held, C. (2010). The interplay between individual and collective knowledge: technologies for organisational learning and knowledge building. Knowledge Management Research \& Practice, 8(1), 33-44.

Kipp, M. E. I., \& Campbell, D. G. (2006). Patterns and Inconsistencies in Collaborative Tagging Systems: An Examination of Tagging Practices. Annual General Meeting of the American Society for Information Science and Technology. Austin, Texas (US).

Lavoué, E. (2011a). Social Tagging to Enhance Collaborative Learning. 10th International Conference on Web-based Learning (ICWL 2011), LNCS 7048, Min, H. Leung et al. (Eds.), (p. 92101). Hong Kong: Springer.

Lavoué, E. (2011b). TaCS : une plate-forme collaborative à base de tags pour l'apprentissage. 5ème Conférence en Environnement Informatique pour l'Apprentissage Humain (EIAH 2011) (p. 163176). Université de Mons, Belgique.

Michlmayr, E., \& Cayzer, S. (2007). Learning user profiles from tagging data and leveraging them for personal(ized) information access. Proceedings of the Workshop on Tagging and Metadata for Social Information Organization, 16th World Wide Web Conference (WWW2007). Banff, Alberta, Canada.

Mika, P. (2007). Ontologies are us: A unified model of social networks and semantics. Journal of Web Semantics, 5(1), 5-15.

Millen, D., Feinberg, J., \& Kerr, B. (2006). Dogear: Social bookmarking in the enterprise. Proceedings of the 24th international conference on Human Factors in computing systems (CHI 2006) (p. 111-120). Montreal, Canada: ACM. 


\section{Author}

Nelson, L., Held, C., Pirolli, P., Hong, L., Schiano, D., \& Chi, E. H. (2009). With a little help from my friends: examining the impact of social annotations in sensemaking tasks. Proceedings of the 27 th international conference on Human factors in computing systems (CHI 2009) (p. 1795-1798). Boston, MA, USA: ACM.

Trant, J. (2009). Studying Social Tagging and Folksonomy: A Review and Framework. Journal of Digital Information, 10(1).

Yeung, C. A., Gibbins, N., \& Shadbolt, N. (2009). Contextualising tags in collaborative tagging systems. Proceedings of the 20th ACM conference on Hypertext and hypermedia (p. 251-260). Torino, Italy: ACM.

Yew, J., Gibson, F. P., \& Teasley, S. (2006). Learning by Tagging: The Role of Social Tagging in Group Knowledge Formation. Learning by Tagging: The Role of Social Tagging in Group Knowledge Formation, 2(4). 
Figure 1. Creation of a document and its assigning tags

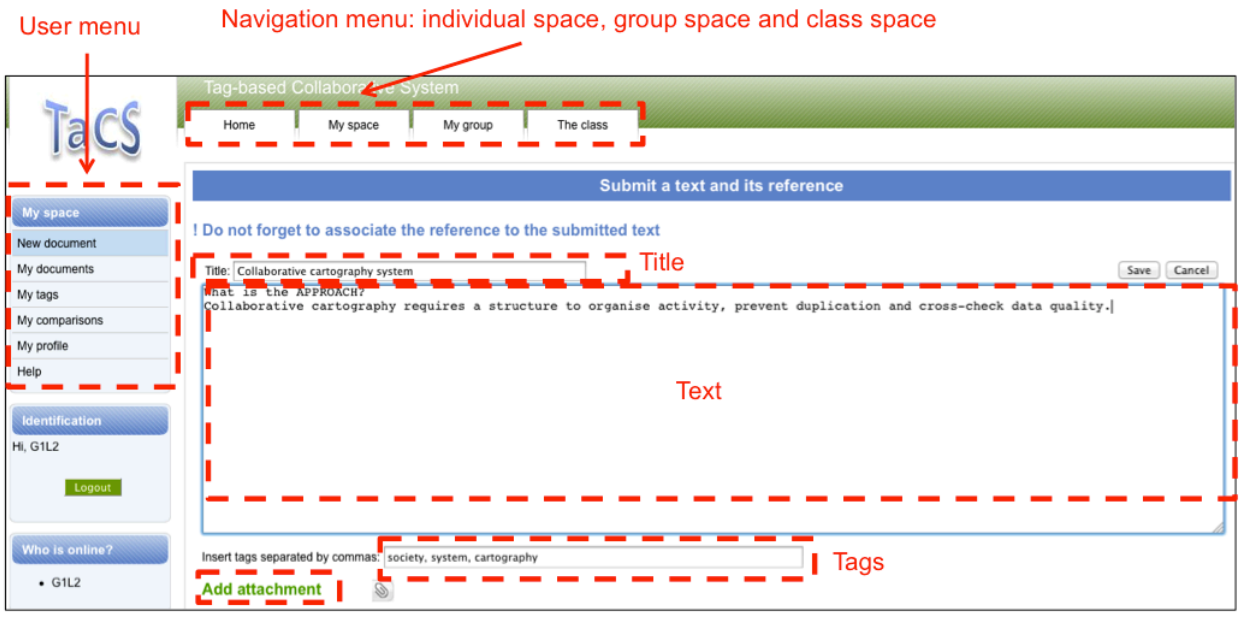

Link to add a reference

Figure 2. Tag clouds of a learner on TaCS (implicit and explicit information)

\section{My tags}

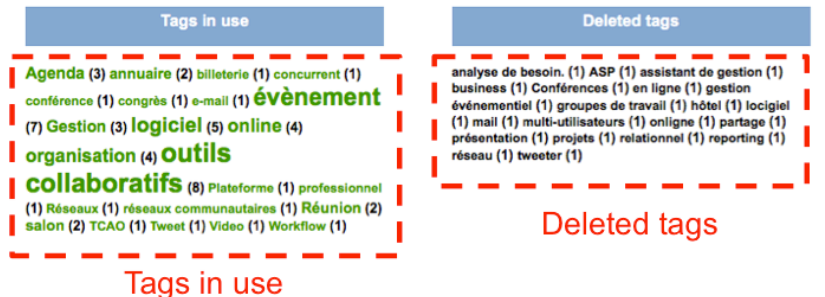

- - - - - - - - - -

| Agenda (1) amlando (1) analysec Co

I nualro (1) blloterie (1) business (2) cas réol (1)

congrès (2) o-moll (1) tuenomentel (1)

évènements (2) hottel (1) locigiol (1) logiciel

| évènements (2) hottel (1) locigiel (1) logiciel
(3) modo do collaboration (1) organisatiton (1) outll (1) |

(1) Salon (3) salons (1) travail collaboratif

(2) Tweet (2) twoetor (1)

- - - -

Tags used to navigate

in the information space 


\section{Author}

Figure 3. Comparison on TaCS of the tag cloud of a student and the tag cloud of his/her group

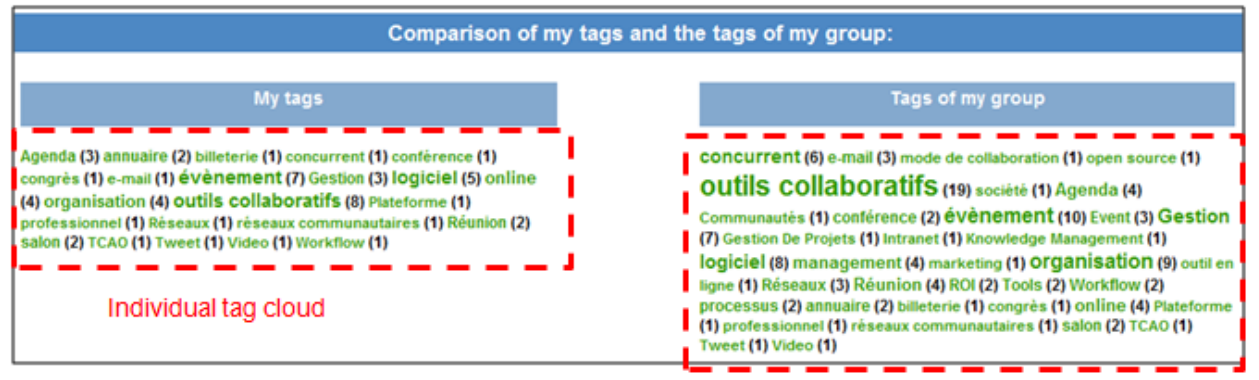

Tag cloud of the group

Figure 4. Details of tags and documents: document-tags, tag-documents and tag-users relationships

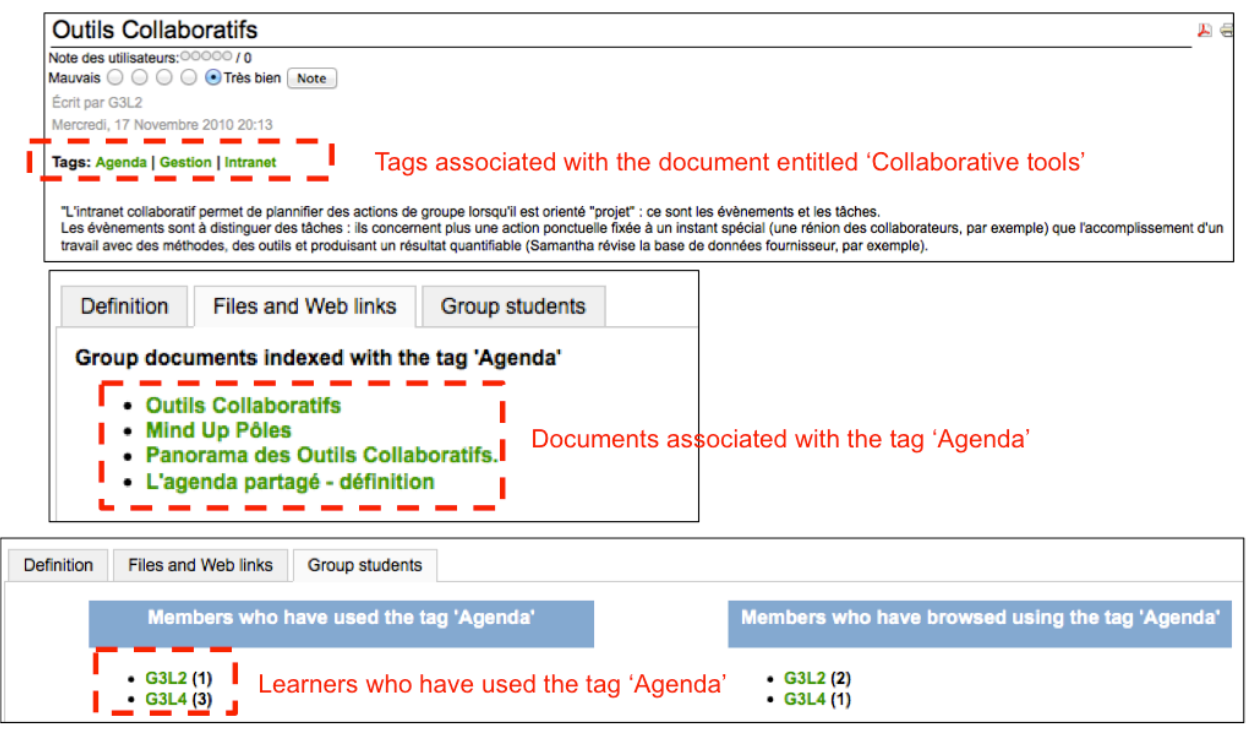


Title

Figure 5. Documents, tags and tag-document relationships created and deleted at each stage of the scenario

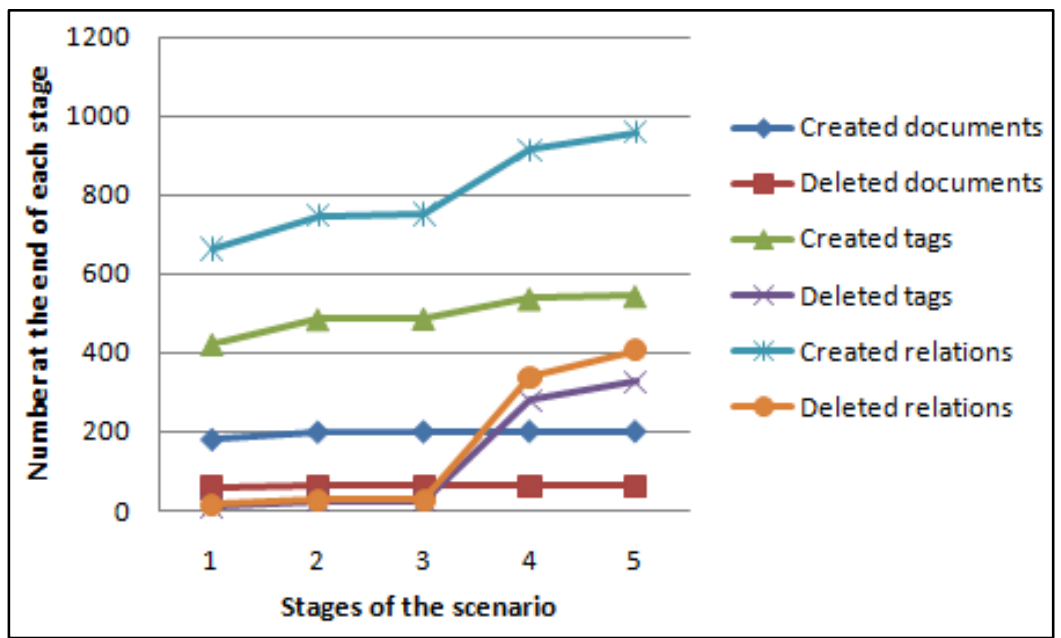

